\title{
Genome-wide identification and transcript profiles of walnut heat stress transcription factor involved in abiotic stress
}

\author{
Xuejiao Liu, Panpan Meng, Guiyan Yang, Mengyan Zhang, Shaobing Peng ${ }^{*}$ and Mei Zhi Zhai
}

\begin{abstract}
Background: Walnut (Juglans regia) is an important tree cultivated worldwide and is exposed to a series of both abiotic and biotic stress during their life-cycles. The heat stress transcription factors (HSFs) play a crucial role in plant response to various stresses by regulating the expression of stress-responsive genes. HSF genes are classified into 3 classes: HSFA, HSFB, and HSFC. HSFA gene has transcriptional activation function and is the main regulator of high temperature-induced gene expression. HSFB gene negatively regulates plant resistance to drought and $\mathrm{NaCl}$. And HSFC gene may be involved in plant response to various stresses. There are some reports about the HSF family in herbaceous plants, however, there are no reports about the HSFs in walnut.

Result: In this study, based on the complete genome sequencing of walnut, the bioinformatics method was used and 29 HSF genes were identified. These HSFs covered 18 HSFA, 9 HSFB, and 2 HSFC genes. Phylogenetic analysis of these HSF proteins along with those from Arabidopsis thaliana showed that the HSFs in the two species are closely related to each other and have different evolutionary processes. The distribution of conserved motifs and the sequence analysis of HSF genes family indicated that the members of the walnut HSFs are highly conserved. Quantitative Real-Time PCR (qRT-PCR) analysis revealed that the most of walnut HSFs were expressed in the walnut varieties of 'Qingxiang' and 'Xiangling' under high temperature (HT), high salt and drought stress, and some JrHSFs expression pattern are different between the two varieties.
\end{abstract}

Conclusion: The complex HSF genes family from walnut was confirmed by genome-wide identification, evolutionary exploration, sequence characterization and expression analysis. This research provides useful information for future studies on the function of the HSF genes and molecular mechanism in plant stress response.

Keywords: Heat stress transcription factors (HSFs), Multiple alignments, Phylogenetic analysis, Motif distribution, Expression profiles

\section{Background}

Walnut (Juglans regia) is an important nut tree cultivated worldwide [1]. In 2017, its planting area was about 489,866 ha, and the output was about 1,925,403 tons in China (FAO, http://www.fao.org/faostat/en/\#data/QC/ visualize). However, the walnut is suffering from both

\footnotetext{
* Correspondence: pshaobing@nwsuaf.edu.cn

Laboratory of Walnut Research Center, College of Forestry, Northwest A \& F University, Yangling 712100, Shaanxi, China
}

abiotic (e.g., high temperature, drought, high salt, chilling.) and biotic (e.g., pathogenic microorganisms and pests) stresses during its life-cycle. In recent years, as "greenhouse effect" has intensified all around the world, high temperature (HT) have reduced the yield of most agricultural and forestry crops to some degree, including the walnut plants. It is generally believed that with a temperature $10^{\circ} \mathrm{C}$ to $15^{\circ} \mathrm{C}$ higher than the ambient temperature, plants would have a heat shock response (HSR) and obtain a heat resistance quickly within a few

(c) The Author(s). 2020 Open Access This article is licensed under a Creative Commons Attribution 4.0 International License, which permits use, sharing, adaptation, distribution and reproduction in any medium or format, as long as you give appropriate credit to the original author(s) and the source, provide a link to the Creative Commons licence, and indicate if changes were made. The images or other third party material in this article are included in the article's Creative Commons licence, unless indicated otherwise in a credit line to the material. If material is not included in the article's Creative Commons licence and your intended use is not permitted by statutory regulation or exceeds the permitted use, you will need to obtain permission directly from the copyright holder. To view a copy of this licence, visit http://creativecommons.org/licenses/by/4.0/ The Creative Commons Public Domain Dedication waiver (http://creativecommons.org/publicdomain/zero/1.0/) applies to the data made available in this article, unless otherwise stated in a credit line to the data. 
hours to withstand the HT which may be lethal [2]. Meanwhile, in China, walnuts are mainly planted in arid and semi-arid regions, where are drought and less rainfall in spring and summer, and precipitation is unevenly distributed. Therefore, moisture is one of the key factors that affect the growth and development of walnuts, as well as the yield and quality of nuts [3]. Moreover, soil salinization and secondary salinization deserve attention. Excessive salt can cause imbalance in osmotic regulation of plants, and excessive accumulation of $\mathrm{Na}^{+}$can also cause ion toxicity. Therefore, the effect of the HT, drought and salt stress on the growth and development of the walnut cannot be ignored.

Gene expression changes triggered by various abiotic stresses are important mechanisms that enable plants to respond and adapt to adverse conditions and thus ensure survival [4]. Therefore, the possible impact of heat, salt and drought stress on walnuts and its molecular mechanisms have been widely surveyed in recent years [5]. Heat stress transcription factors (HSFs) is a protein with transcriptional regulatory activity that responds to a variety of stresses [6]. HSF proteins have five typical structural features: a highly conserved DNA-binding domain (DBD) at the N-terminus, an oligomerization domain (OD or HR-A/B), a nuclear localization signal (NLS), a nuclear export signal (NES) and a C-terminal domain (CTD) at the C-terminus [7]. The DBD domain of HSFs can accurately recognize the heat shock element (HSE: 5'-AGAAnnTTCT-3'; n: any base) that located in the upstream promoter region of heat shock proteins (HSPS) genes and induce the transcription of HSP genes $[8,9]$. Depending on the number of amino acids that inserted between the A and B segments of the HR-A/B region, $H S F$ genes are classified into 3 classes: $H S F A$, $H S F B$, and HSFC. The structure of the class $H S F B$ is relatively simple and has no amino acid inserted between the $\mathrm{A}$ and $\mathrm{B}$ segments, whereas the classes HSFA and HSFC have 21 and 7 amino acids inserted between the $\mathrm{A}$ and $\mathrm{B}$ segments, respectively [10]. The CTD of most class HSFA is acidic and contains short peptide motifs (acidic amino acid residues, AHA: Activator motifs) with central Trp or Phe residues [11, 12]. The AHA motifs are essential for activation function, and a similar motif has also been identified as part of the activation region of transcription factors (TFs) in mammalian and yeast (Saccharomyces cerevisiae) [12, 13]. HSFB and HSFC lack the AHA motifs; and therefore, they are considered to have no transcriptional activation function. Under normal conditions, HSFs exists in the cytoplasm and nucleoplasm without the activity binding to DNA. HSP70 (or HSP90 and multi-companion complex) interacts with HSFs to make HSFs in a passivated monomer state. However, abnormal proteins will be produced during heat shock and then HSPs can be deprived from HSFs and release HSFs, further, HSFs in the nucleus will be assembled into a trimer that binds to the thermal response element at the $5^{\prime}$ end of the heat shock to induce transcription [14].

Although the adversity response function of HSF genes is not well understood in most plants, the information about the HSFs has accumulated in A. thaliana and Lycopersicon esculentum. For example, in L. esculentum, HSFA1a has been found to be a major regulator for the induction of heat-resistant genes and synthesis of HSFA2 and HSFB1 $[15,16]$; The expression of HSFA1, HSFA2 and HSFB1 were affected by salicylic acid (SA) under heat shock conditions [17]. In A. thaliana, HSF1, HSF3, HSFA 2 and HSFA3 are related to heat tolerance, and HSFA 2 is the most strongly induced one by heat; overexpression of HSFA2 not only enhances plants with basic and heat resistance, but also improves the tolerance of root callus; When osmotic stress occurs, HSFA2 mutations lead to a significant reduction in basic heat tolerance and antioxidant capacity [18]. Due to the extensive multifaceted roles in anti-stress response, the HSFs has recently attracted broad attentions. However, there were few reports on walnut HSFs. Considering that abiotic stress causes a significant reduction in walnut yield, and HSFS plays a non-negligible role in plant stress resistance, a better understanding of the function of walnut HSF genes is important. In this study, the walnut HSFs was identified and analyzed according to the released genome [1]. Phylogenetic tree analysis revealed that the evolutionary relationships of HSFs between walnut and A. thaliana are different. Quantitative Real-Time PCR (qRT-PCR) analysis provided a solid basis for further functional characterization of the HSF genes. In addition, the results may provide vital information for understanding the walnut adversity adaptation mechanism, which will benefit for walnut industry.

\section{Results \\ Genome-wide identification and chromosomal locations of walnut HSF genes}

Total 33 candidate HSF genes were identified by BLAST and HMMER methods. Among the 33 candidates, 4 sequences were repeated and abandoned. Finally, 29 walnut HSF genes were confirmed and named from JrHSF01 to JrHSF29. The molecular weight of these HSF proteins is between $14.43 \mathrm{kDa}$ and $65.42 \mathrm{kDa}$, consisting with $128 \sim 505$ amino acid residues. The theoretical PIs of these JrHSFs are $2.12 \sim 9.28$ (Table 1 ).

These 29 JrHSF genes were located on 13 chromosomes of $J$. regia, while the chromosomes 4S, 7D and 8S do not contain any JrHSF gene. There are 4 JrHSF genes mapped on the chromosome 2D, which contains the most number of JrHSF genes. The chromosomes 2S, 4D, $6 \mathrm{D}$ and $7 \mathrm{~S}$ each contain only 1 JrHSF gene (Fig. 1). 
Table 1 The HSF genes in J. regia

\begin{tabular}{|c|c|c|c|c|c|c|}
\hline Gene & Accession No. & Gene ID & Chromos-ome & $\begin{array}{l}\text { Number of } \\
\text { amino acids/aa }\end{array}$ & $\begin{array}{l}\text { Molecular } \\
\text { weight/kDa }\end{array}$ & Theoretical PI \\
\hline JrHSFO1 & XP_018829016.1 & LOC108997276 & Chr3S & 500 & 55.64 & 5.12 \\
\hline JrHSFO2 & XP_018811267.1 & LOC108983931 & Chr1D & 277 & 30.62 & 5.26 \\
\hline JrHSFO3 & XP_018845450.1 & LOC109009449 & Chr2D & 480 & 53.69 & 4.89 \\
\hline JrHSFO4 & XP_018845303.1 & LOC109009313 & Chr2D & 471 & 52.78 & 4.84 \\
\hline JrHSF05 & XP_018829017 & LOC108997276 & Chr3S & 500 & 55.64 & 2.12 \\
\hline JrHSFO6 & XP_018856444 & LOC109018727 & Chr4D & 363 & 41.39 & 5.69 \\
\hline JrHSFOT & XP_018830717 & LOC108998591 & Chr2D & 300 & 33.45 & 6.26 \\
\hline JrHSFO8 & XP_018839407 & LOC109005079 & Chris & 368 & 42.22 & 4.96 \\
\hline JrHSFO9 & XP_018849985 & LOC109012680 & Chr5D & 390 & 44.47 & 5.26 \\
\hline JrHSF1O & XP_018844061.1 & LOC109008434 & Chr1D & 505 & 65.42 & 5.75 \\
\hline JrHSF11 & XP_018818650 & LOC108989484 & Chr1D & 321 & 37.37 & 5.58 \\
\hline JrHSF12 & XP_018847363 & LOC109010870 & Chr3D & 128 & 14.43 & 8.8 \\
\hline JrHSF13 & XP_018848541.1 & LOC109011701 & Chris & 490 & 54.58 & 5.58 \\
\hline JrHSF14 & XP_018805575.1 & LOC108979361 & Chr2D & 336 & 38 & 5.49 \\
\hline JrHSF15 & XP_018813737.1 & LOC108985770 & Chris & 277 & 30.71 & 6.23 \\
\hline JrHSF16 & XP_018838842.1 & LOC109004663 & Chr2S & 332 & 37.52 & 5.59 \\
\hline JrHSF17 & XP_018811948.1 & LOC108984436 & Chr6D & 344 & 37.28 & 4.82 \\
\hline JrHSF18 & XP_018847277.1 & LOC109010817 & Chr3D & 505 & 55.39 & 5.02 \\
\hline JrHSF19 & XP_018820155 & LOC108990606 & Chr5D & 206 & 23.63 & 9.28 \\
\hline JrHSF2O & XP_018822295 & LOC108992254 & Chr8D & 363 & 40.65 & 8.16 \\
\hline JrHSF21 & XP_018839503 & LOC109005155 & Chr5s & 503 & 56.92 & 5.14 \\
\hline JrHSF22 & XP_018848321.1 & LOC109011524 & Chr5s & 208 & 24.25 & 8.5 \\
\hline JrHSF23 & XP_018805848.1 & LOC108979602 & Chr5s & 390 & 44.34 & 5.05 \\
\hline JrHSF24 & XP_018818420.1 & LOC108989320 & Chr5D & 250 & 28.54 & 6.97 \\
\hline JrHSF25 & XP_018855724.1 & LOC109017997 & Chr7s & 287 & 33.17 & 6.46 \\
\hline JrHSF26 & XP_018836499.1 & LOC109003006 & Chr3D & 351 & 40.31 & 4.78 \\
\hline JrHSF27 & XP_018809115.1 & LOC108982254 & Chr6s & 497 & 55.65 & 4.84 \\
\hline JrHSF28 & XP_018858526.1 & LOC109020508 & Chr8D & 383 & 42.96 & 4.69 \\
\hline JrHSF29 & XP_018811530.1 & LOC108984137 & Chr6s & 489 & 54.72 & 4.84 \\
\hline
\end{tabular}

\section{The conservative domains of JrHSFs}

The JrHSFs protein sequences were aligned and the result showed that the DBD domain exists in all JrHSF sequences and is highly conserved. The number of amino acid residues is from 94 (JrHSF12) to 103 (JrHSF19) (Fig. 2a). However, there are different degrees of insertion or deletion in these proteins. For example, 9 amino acids are inserted between $\alpha 1$ and $\beta 1$ in JrHSF19, 6 amino acid sequences are inserted between $\alpha 1$ and $\beta 1$ in JrHSF22, however, 36 amino acid residues are lacked in JrHSF12, who delete 3 amino acid residues between $\alpha 2$ and $\alpha 3$, and 33 amino acid residues between $\beta 3$ and $\beta 4$ (Fig. 2b).

Sequence logos were constructed using WebLogo program and showed that the HSF domain is highly-conserved with $100 \%$ of amino acids sequence identity at sites of $1,9,14,15,18$, 22, 25, 26, 28, 29, 30, 31, 33, 34, 35, 45, 50, and so on (Fig. 2c).

\section{Evolutionary relationship of the JrHSF genes}

An un-rooted phylogenetic tree relating to the evolutionary relationship between the HSFs from the walnut and Arabidopsis was constructed (Fig. 3). According to the classification of $A$. thaliana, the HSFs of these two plants was divided into three groups: group A contains $18 \mathrm{JrHSFs}$, group B contains $9 \mathrm{JrHSFs}$, and group C contains 2 JrHSFs. The group A was further divided into 9 subgroups (A1 to A9), of which A5 contains only AT4G13980 (HSFA5), A7 includes only AT3G51910 (HSFA7), and A9 covers only AT5G54070 (HSFA9). Meanwhile, no Orthologous and paralogous HSF genes from walnut were found in the above three subgroups, suggesting that gene deletions may have occurred during evolution. The group B was divided into four subgroups (B1 to B4), of which B1 contains only AT4G36990 


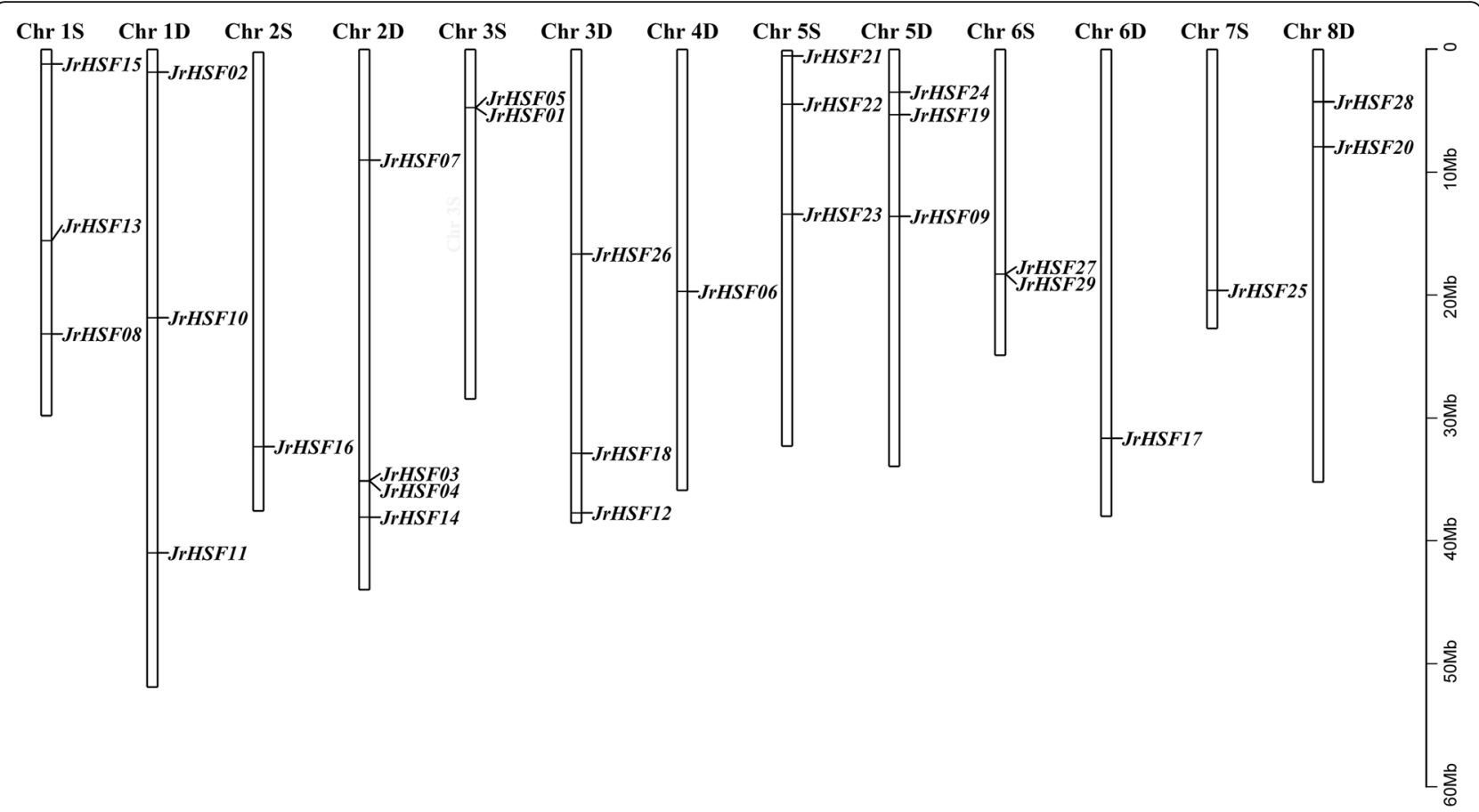

Fig. 1 Distribution of the JrHSF genes on pseudo chromosomes of J. regia. The scale on the right is in million bases (Mb). D: Dominant; S: Subdominant

(HSFB1) with no homologous from. The group $\mathrm{C}$ was not further divided (Fig. 3).

\section{Conservative motif distribution and sequence feature of the JrHSFs}

The MEME was used to analyze the motifs in the JrHSFs and their basic information (Table 2 and Fig. 4). The results showed that there are 20 different conserved motifs (including $11 \sim 50$ amino acids) in these $29 \mathrm{JrHSFs}$, and each JrHSF include 2 16 conserved motifs (Table 2, Fig. 4). Among 29 JrHSFs, JrHSF01, JrHSF05 and JrHSF22 have the most number of conserved motifs, while JrHSF16 contains only 2 conserved motifs; JrHSF23, JrHSF24 and JrHSF26 contain 3 conserved motifs; JrHSF07, JrHSF21, JrHSF28 and JrHSF29 contain 4 conserved motifs, respectively. Furthermore, there are 4 motifs (Motif1, Motif2, Motif3 and Motif4) are completely conserved in these 29 JrHSFs. Most of the 29 JrHSFs have Motif1, Motif2 and Motif3. The Motif1 (FVVWBPPEFARDLLPKYFKHNNFSSFVR QLNTYGFRKVDPDRWEFANEGF) is located in $\beta 2-\beta 4$. The Motif2 (PFLTKTYDMVDDPATDSIVSW) is located in $\alpha 1-\beta 1$. The Motif3 is dispersed in the DBD and is highly conserved (Fig. 4). The Motifs 1-3 represent the DBD domain. Therefore, it is concluded that the members of the JrHSFS are highly conserved.

\section{Expression of the JrHSFs in the walnut}

The expression of JrHSFs was analyzed in the leaves of the 'Qingxiang' and 'Xiangling' using qRT-PCR under drought, HT and high salt stresses. The results showed that all JrHSFs were expressed under these stresses with different expression patterns (Fig. 5).

In 'Qingxiang'. (1) Under drought stress, the expression of eight JrHSFS (JrHSF24, JrHSF13, JrHSF19, JrHSF15, JrHSF11, JrHSF22 and JrHSF09) were increased at $7 \mathrm{~d}$ and then decreased at $22 \mathrm{~d}$; the expression levels of JrHSF08, JrHSF2O and JrHSFO3 were enhanced at 13 d; five JrHSFs (JrHSF16, JrHSF24, JrHSF15, JrHSF11 and JrHSF05) maintained high expression after $7 \mathrm{~d}$, but low transcription after rehydration. (2) After heat stress, the transcription of JrHSF21, JrHSF13 and JrHSFO6 reached to peak level at $8 \mathrm{~h}$; five other JrHSFS (JrHSF28, JrHSF17, JrHSF19, JrHSF08 and JrHSF11) increased to maximum level at 24h; and eight JrHSFs (JrHSF21, JrHSF13, JrHSF06, JrHSF17, JrHSF02, JrHSF19, JrHSF08 and JrHSF15) maintained high expression status after $2 \mathrm{~h}$. (3) Under salt stress, thirteen JrHSFs (JrHSF13, JrHSF17, JrHSF19, JrHSF8, JrHSF15, JrHSF26, JrHSF18, JrHSF23, JrHSF10, JrHSF05, JrHSF01, JrHSF22 and JrHSF09) showed the highest abundance at $24 \mathrm{~h}$ and then decreased. In summary, the expression of JrHSF28, JrHSF21,JrHSF06, JrHSFO2 and JrHSFO7 under heat 


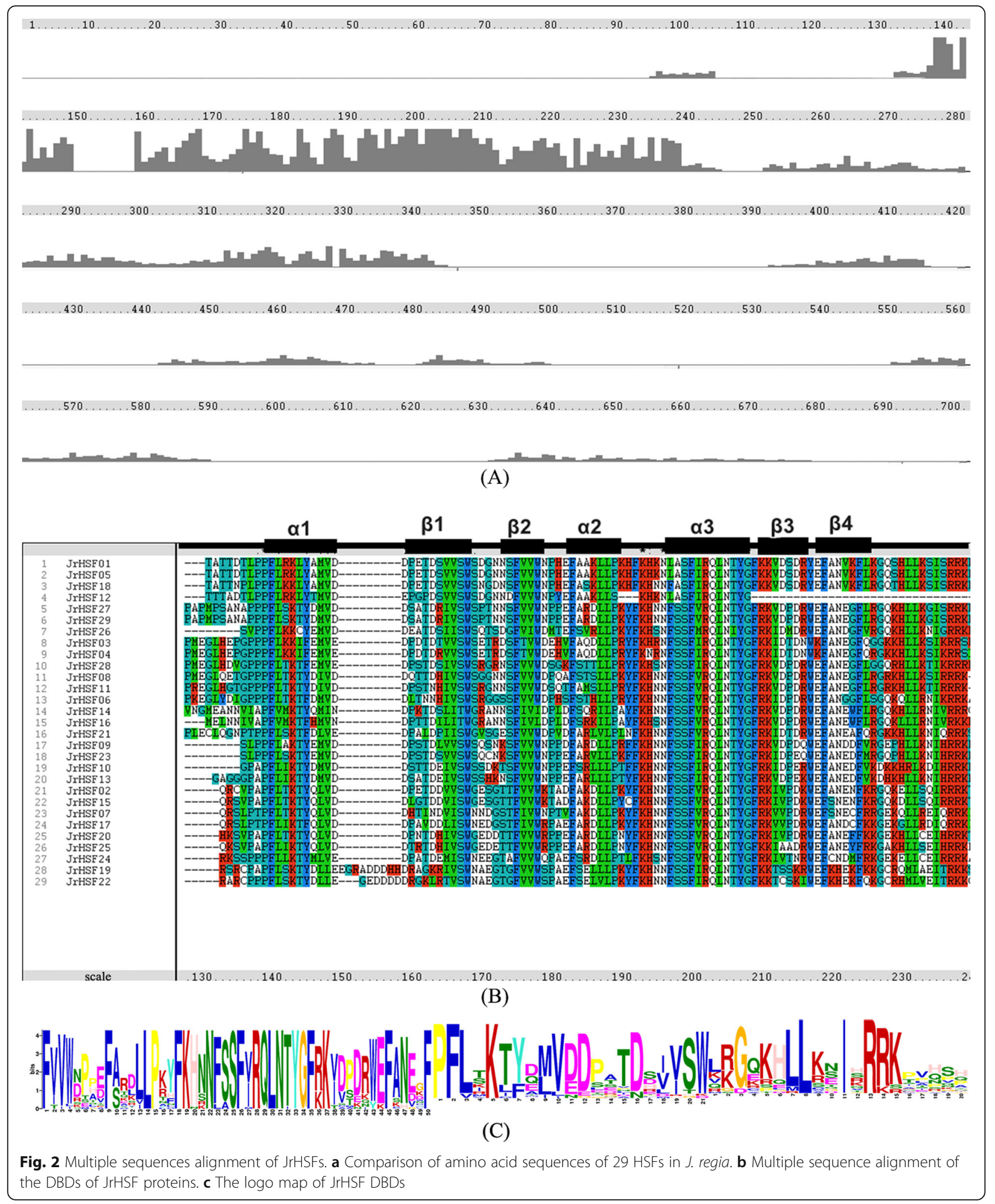

stress was significantly higher than that under drought and salt stresses, but the expression patterns of JrHSFO5 and JrHSFO1 was opposite. In addition, JrHSF13 and
JrHSF17 maintained high expression levels while JrHSF25, JrHSF04 and JrHSF12 were hardly expressed under above three stresses (Fig. 5). 


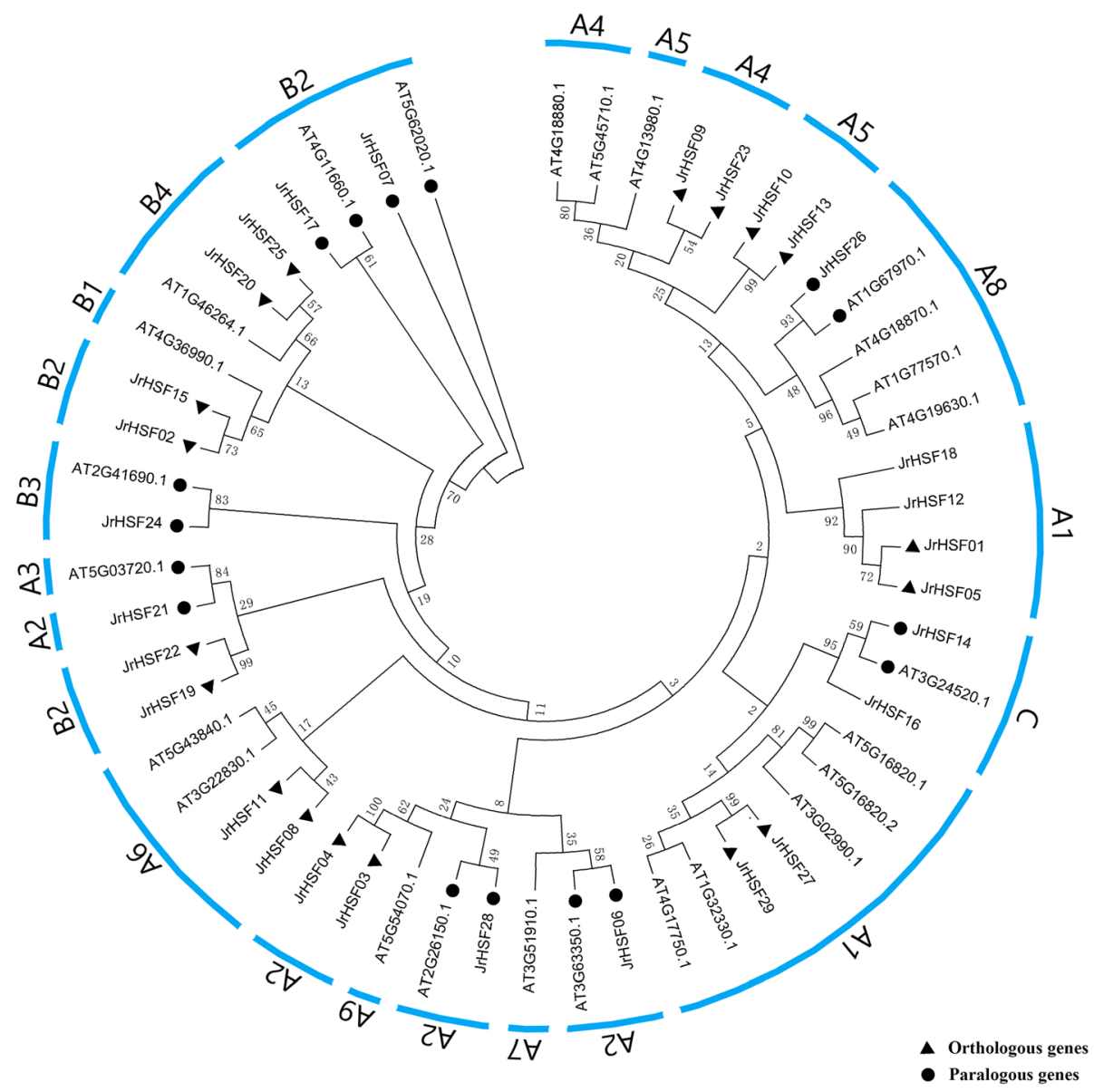

Fig. 3 Phylogenetic tree analysis of the HSFs from J. regia (Jr) and Arabidopsis (AT). Orthologous and paralogous genes were indicated by circle and roundness, respectively

In 'Xiangling'. (1) Under drought stress, the expression level of JrHSF2O reached to peak level at $22 \mathrm{~d}$, most of the JrHSFs were hardly expressed after rehydration. (2) After heat stress, JrHSF22 maintained a high expression level from $0 \mathrm{~h}$ to $24 \mathrm{~h}$; the expression of nine JrHSFs (JrHSF28, JrHSF21, JrHSF08, JrHSF17, JrHSF06, JrHSF02, JrHSF07, JrHSF19 and JrHSF15) were increased significantly at $8 \mathrm{~h}$. (3) Under salt stress, five JrHSFs (JrHSF22, JrHSF21, JrHSF08, JrHSF19 and JrHSF20) reached to maximum value at $12 \mathrm{~h}$, while the others displayed low expression levels. In general, JrHSF22, JrHSF08, JrHSF19 and JrHSF20 maintained high expression levels while JrHSF25 and JrHSFO4 were hardly expressed under above three stresses (Fig. 5).

In short, most of JrHSFs responded to HT, high salt and drought stresses in walnut, the expression pattern of most JrHSFs was different between 'Qingxiang' and 'Xiangling'.

\section{Discussion}

The walnut HSF genes displayed diverse characters A phylogenetic tree was constructed with 29 HSF proteins from J. regia and 25 HSF proteins from A. thaliana (Fig. 3).
Nine pairs of orthologous genes and eight pairs of paralogous genes were found, and the paralogous genes of $A$. thaliana HSFs AT4G18870 and AT5G54070 were not found in walnut, indicating that most HSF members are specific for reproductive isolation in different species. This phenomenon has also been found in other plant gene families. In Arabidopsis and rice (Oryza sativa), for instance, most wall-associated kinase (WAK) genes have speciesspecific expansion [19]. The lineage-specific divergence of nucleotide binding site-leucine-rich repeat (NBS-LRR) genes may occur to enable plant response to pathogens unique to each species [20]. Small auxin-up RNAs (SAURs) genes were clustered in species-specific distinct clades and expanded in a species-specific manner [21]. Moreover, Duan et al. [5] believed that HSFC may have different functions in the wheat (Triticum aestivum), because TaHsfC3 has no orthologous genes in the rice, Arabidopsis and maize (Z. mays) HSF families. In grape (Vitis vinifera), Homeobox $(H B)$ genes were not found in three subgroups (PLINC, NDX or LDof) of Arabidopsis, $\mathrm{Li}$ et al. [22] revealed that $H B$ genes may have been 
Table 2 Motif sequences identified by MEME tool

\begin{tabular}{|c|c|c|}
\hline Motif & $\begin{array}{l}\text { Number of } \\
\text { amino acids }\end{array}$ & Best possible match \\
\hline Motif1 & 50 & FWWBPPEFARDLLPKYFKHNNFSSFVRQLNTYGFRKVDPDRWEFANEGF \\
\hline Motif2 & 21 & PFLTKTYDMVDDPATDSIVSW \\
\hline Motif3 & 20 & LRGQKHLLKNIHRRKPVHSH \\
\hline Motif4 & 29 & MEQRQQQMMSFLAKAMQNPGFJAQLVQQQ \\
\hline Motif5 & 29 & FGLEEEIERLKRDKNVLMQELVKLRQQQZ \\
\hline Motif6 & 30 & APVPPGINDTFWEQFLTETPGTSDADEISS \\
\hline Motif7 & 41 & EETILPEFSEJQGIMPESTAEIPDMNYAGSETGNASYVDPM \\
\hline Motif8 & 32 & PSMNEAAKALLRQILKMBGSPRVEPLMBNPGA \\
\hline Motif9 & 29 & HGLZQGKKNGWDKIQHMDKLTEQMGLLAS \\
\hline Motif10 & 50 & PISAELFMPAEPEFPISSPSTANSDIQSSSYAMPDHAIEAQFPNLDVYNS \\
\hline Motif11 & 21 & NRRITAGNKKRRLPIEEESES \\
\hline Motif12 & 11 & SSLGACVEVGK \\
\hline Motif13 & 15 & PPPQPMEGLHETGPP \\
\hline Motif14 & 29 & JFDDAPSTNAFDSGSSTNRVSGVTLSEVP \\
\hline Motif15 & 15 & ATDHQLQAMEQRLQG \\
\hline Motif16 & 11 & NAPDGQIVKYQ \\
\hline Motif17 & 15 & MADVNDAGSSTTATT \\
\hline Motif18 & 50 & VNGSLPIEIDYISPDADIDLFLSDPNFWDDLVQSPVPEDIESNSVQGMSK \\
\hline Motif19 & 29 & KFGSDQEDLIVKQGDCGGSRGGLVEQAGG \\
\hline Motif20 & 48 & KQQKRELDGEEFVKRRRLLASHGREKAIDKIHRINCRNQVPGGLVTTL \\
\hline
\end{tabular}

deleted during evolution. Although the apple (Malus domestica) WAKY genes has a small number of mutations compared to the Arabidopsis WAKY genes, it also shows that the plant $W A K Y$ genes family evolved in diversity [23]. All these findings suggested that they had undergone varying degrees of species-specific changes, and these JrHSFs have abundant characters.

\section{JrHSFs gene expression patterns responded to abiotic stresses implied multiple roles}

JrHSFs displayed various expression patterns in one walnut cultivar exposed to HT, drought and high salt stresses, and also showed diverse transcription profiles in 'Qingxiang' and 'Xiangling' under each of above three treatments (Fig. 5), for instance, under HT stress, in 'Xiangling', JrHSF13 had the largest change in expression, while in 'Qingxiang', JrHSF22 showed the largest change in transcription; JrHSFO2, JrHSFO6, JrHSFO8, JrHSF17, JrHSF19 and JrHSF21 all revealed changing expression profiles in either 'Qingxiang' or 'Xiangling'; The expression levels of JrHSF04, JrHSF12 and JrHSF25 were generally low. Interestingly, different types of JrHSFs were expressed differently, which is similar to other species. For example, HSFA1 members of JrHSF01, JrHSF05, JrHSF12, JrHSF18 and JrHSF27 show obvious expression under salt stress, which is consistent with the findings observed by Duan et al. [5], who believed that the mutant strain of HSFA1a was highly sensitive to salt stress, and all HSFA1 were involved in osmotic stress response. JrHSFO2 belongs to HSFB and had a higher expression level under heat stress, while hardly expressed under drought and salt stresses. This result is consistent with the findings of $\mathrm{Li}$ et al. [24] that the rice OsHSF2 $b$ gene negatively regulates plant resistance to drought and salt; and the negative regulation of OsHSF2b is mediated by its C-terminal DBD domain. HSFCs including JrHSF14 and JrHSF16 have relatively low expression levels under three stresses, and the function of HSFC needs further study. These results indicated potential abundant response mechanism for different cultivars exposed to the same stimulus and for different HSF members in the same variety under a stress.

Besides, the response of JrHSFO8 under HT stress showed an up-regulation trend with treatment time prolong in both varieties (Fig. 5). JrHSFO8 belongs to HSFA6, indicating that HSFA may play a key role in resisting abiotic stress, which is consistent with the findings of Liu et al. [25]. JrHSF04 and JrHSF25 were not significant in response to stress. This result may indicate that these genes are less abundant in walnuts. JrHSF13, JrHSF12, JrHSF11, JrHSF10, and JrHSF9 were significantly different in 'Qingxiang' and 'Xiangling', and their 


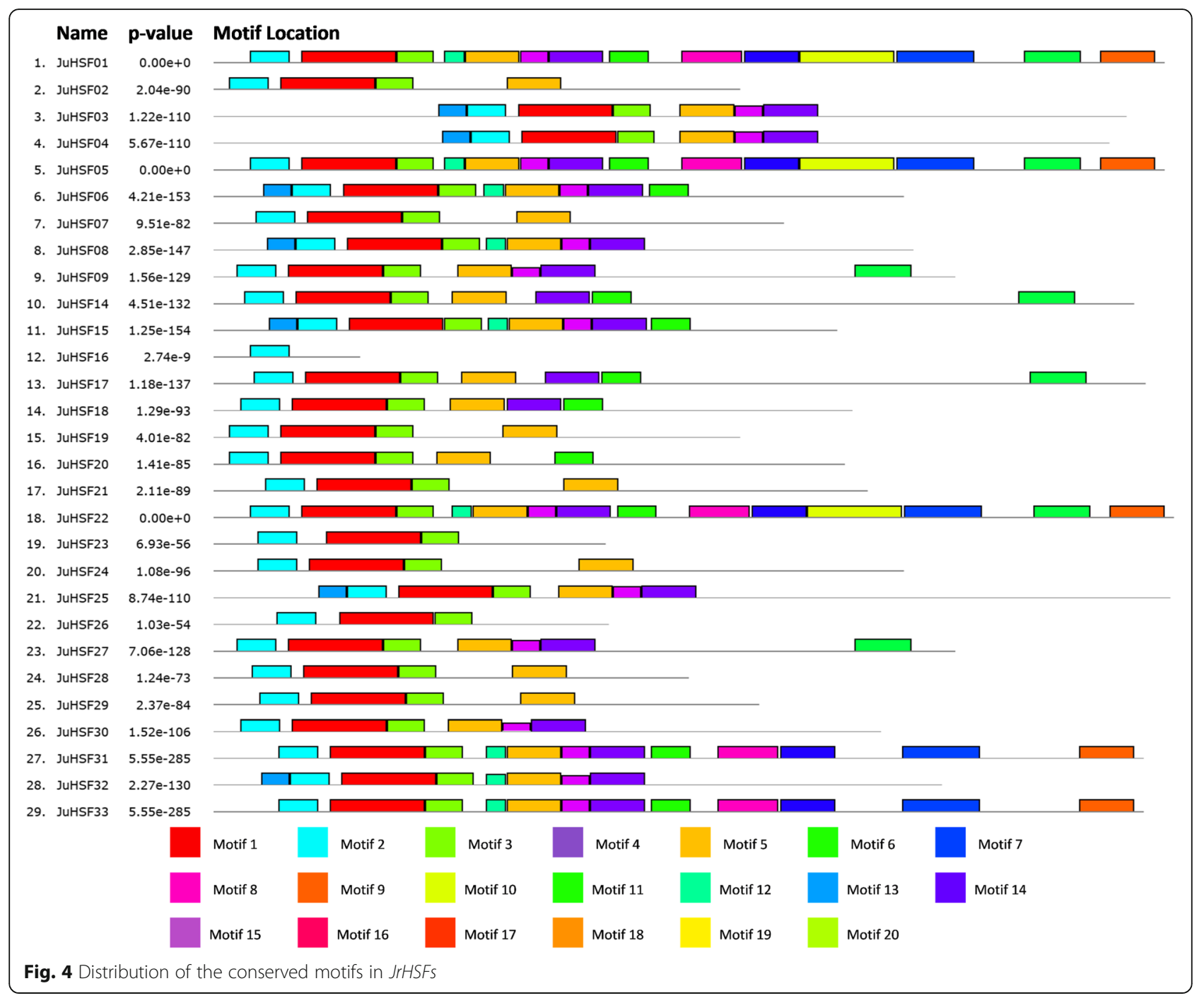

expression levels in 'Qingxiang' were higher than in 'Xiangling'. The reason why late mature walnut is more resistant than precocious walnut may be related to these genes [26]. JrHSF22 had a stronger response to drought and HT stress in 'Xiangling' than in 'Qingxiang', but it was rarely expressed in 'Qingxiang' under salt stress. $J r H S F 22$ belongs to HSFB2, and HSFB may negatively regulate plants to respond to salt stress, which is consistent with the results from Scharf et al. [8]. Meanwhile, the time when the gene expression reaches to the peak was various, implying the initiation of the JrHSFs expression may be different. All these findings suggested that the concrete role of every HSF gene is plant-specific to special conditions.

Moreover, most of the JrHSFs show higher expression levels under the three stresses in 'Qingxiang' than those in 'Xiangling'. This result was also observed in grape varieties 'Jingxiu' and 'Tangwei'. What's more, Liu et al. [25] used this result to predict the potential stress resistant ability of 'Jingxiu' and 'Tangwei'. Therefore, we may also conclude that 'Qingxiang' is more resistant to abiotic stress than 'Xiangling', which further confirmed the fact that 'Qingxiang' is more tolerant to adversity than 'Xiangling'. Meanwhile, HSF genes may be functioned as resistance markers to distinguish different plants responding to the same stress.

Potential regulation of HSFs in heat temperature, drought and salt stresses

Plants can resist the HT stress by producing hormones and osmotic substances. For example, SA pathway can mediate the system-acquired heat resistance (SAR). SA promotes the production of HSPs, and also effectively removes $\mathrm{H}_{2} \mathrm{O}_{2}$ from cucumber [27]. HSPs can increase the denaturation temperature of proteins in plants to protect them from HT damage, and repair the damaged proteins to resist the high temperatures. The expression of HSPs is mainly regulated by the HSFs at the 


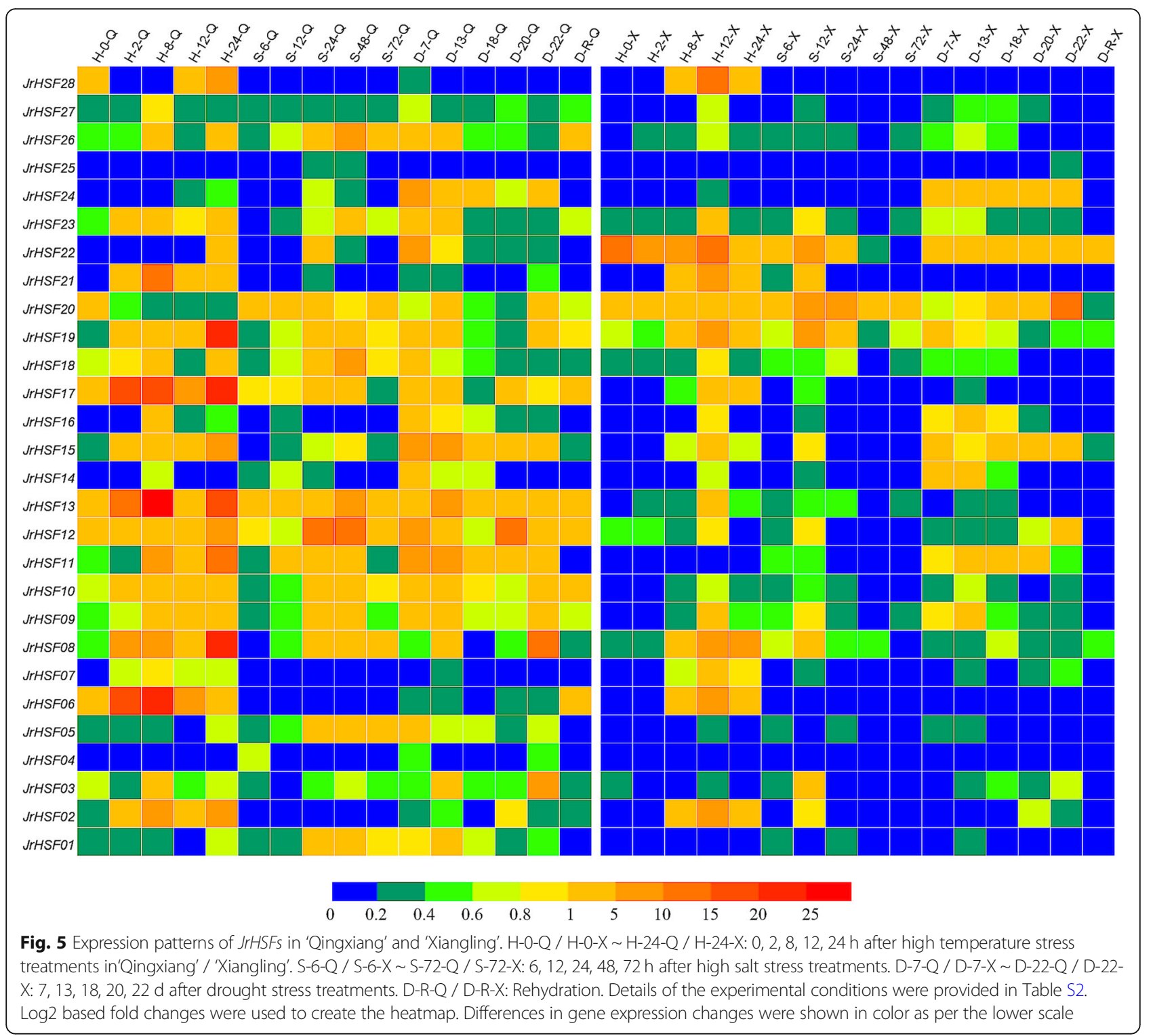

transcriptional level [28]. Like the mammalian HSFs, in plants, the binding of a chaperone to a misfolded protein forces the HSFs to be released to activate expression of the thermosensitive genes. Meanwhile, HSF is a highly conserved central regulator of the HSR in eukaryotes [29]. For example, HSFA2 is accumulated under the HT stress and functioned on signal amplification [30]. $H S F B 2 b$ gene is required for obtaining heat resistance in plants [24]. Rice OsHSFC1a can respond to early HS treatment [29].

Moreover, the role of the HSF signaling pathway is not only limited to thermal stress responses, but also involves in a variety of stresses such as cold, infiltration, drought, salt, ultraviolet light, oxidation and pathogen infection [31]. In the field, the drought stress usually occurs simultaneously with the HT stress. A. thaliana
Galactinol synthase (GolS1) is an important compound produced by plants in response to the drought stress, whose expression is regulated by HT; meanwhile, target GolS1 could mediate the HSFs in plant response to the drought stress [32]. In addition, the HSFs can also act on the downstream drought response element binding factors (DREB2A and DREB2C) to improve the expression of some drought-stress-response genes [33]. Similarly, when subjected to the salt stress, plants are actually subjected to the osmotic stress. This osmotic stress signal can cause the accumulation of abscisic acid (ABA), which further induces the adaptive response of plants [34]. In A. thaliana, the promoter of AtHSFA6a contains two AREs (ABA response elements) and binds to three ABA-responsive TFs in vitro; the plants overexpressing AtHSFA6a exhibit both salt and drought 
tolerance [28]. Peng et al. [35] showed that in grapes the expression of the HSFs is induced by the pathogen infection, HTs and drought, suggesting that the HSFs are associated with both biotic and abiotic stresses in grapes. Interestingly, salt damage, drought stress, and other osmotic stress lead to ROS (Reactive Oxygen Species) accumulation and cell wall changes. Cell wall contains protein and $\mathrm{Ca}^{2+}$ ions [36]. The salt stress can also cause loss of $\mathrm{Ca}^{2+}$ in the cell wall. $\mathrm{Ca}^{2+}$ and $\mathrm{ROS}$ are key factors in the process of evoking the HSR [37-40]. Therefore, it draws our notion that JrHSF genes response to stresses may involve in HSR and ABA signal pathway. To verify this point, further research is needed. Although it is not clear further principles of the HSF genes responding to the HT, salt and drought stresses, this research has shown that the HSF genes do participate in resistance to the HT, drought and salt stresses in the walnut.

\section{Conclusions}

In this study, 29 JrHSFs were identified and their distribution on chromosomes, gene structure, conserved motifs, and evolutionary relationships were analyzed, and found that the JrHSFs are highly conserved and displayed different evolutionary processes from AtHSFs. JrHSF17, JrHSF13,JrHSF12,JrHSF11,JrHSF10, and JrHSF9 were significantly induced and different in both cultivars, implying the necessary attention and further exploration. The various expression of JrHSFs in 'Qingxiang' and 'Xiangling', suggested that JrHSFs may play an important role in plant resistance to high temperature, drought and salt stresses. This research provides useful information for future studies on the function and response mechanism of JrHSF genes.

\section{Methods}

\section{Plant materials}

\section{Seedling preparation}

The tissues used in this experiment were collected from 2year-old J. regia varieties 'Qingxiang' (a late bearing walnut variety) and 'Xiangling' (a precocious walnut variety), which were preserved in a plant factory of Northwest Agriculture \& Forestry University (our lab, in Yangling, Shaanxi province, China) and stored in the Liaoning Economic Forest Research Institute (in Dalian, Liaoning province, China). New branches were obtained and grafted into walnut rootstocks $(J$. regia) which were planted in the mixed turf peat and sand $(2: 1 \mathrm{v} / \mathrm{v})$ in plastic pots in a greenhouse $\left(22 \pm 2{ }^{\circ} \mathrm{C}\right.$, relative humidity $70 \pm 5 \%, 14 \mathrm{~h}$ light/ $10 \mathrm{~h}$ dark). When the height of the walnut seedlings reached to about $55-65 \mathrm{~cm}$, seedlings with similar height and biomass were selected for further experiments. The collected leaf samples were frozen in liquid nitrogen immediately and stored at $-80^{\circ} \mathrm{C}$ for RNA extraction $[41,42]$.

\section{High temperature treatment}

The selected 24 seedlings (12 'Qingxiang' walnuts and 12 'Xiangling' walnuts) were heated $\left(42^{\circ} \mathrm{C}\right)$ for $2 \mathrm{~h}$ in a greenhouse [5]. The leaves were collected at $0,2,8,12$ and $24 \mathrm{~h}$ after heating in triplicate, respectively. Three seedlings of each variety grew normally at room temperature $\left(25^{\circ} \mathrm{C}\right)$ as a control.

\section{Drought stress treatment}

First, the soil moisture of the selected 30 seedlings (15 'Qingxiang' walnuts and 15 'Xiangling' walnuts) was maintained at $60 \%$ for 7 days, and then the watering of 9 seedling of each variety was stopped to reduce the soil moisture naturally. When soil moisture reached $60 \%$ (7 d), $40 \%$ (13 d), 20\% (18 d), 10\% (20 d) and 5\% (22 d), the leaves were collected in triplicate, respectively. Three seedlings of each variety were re-watered. Samplings were performed when soil moisture was increased to $60 \%$ and kept for 3 days (REW60\%). The other 3 seedlings of each variety under normal watering were used as controls. The soil moisture content of each plant was monitored and adjusted by weighing methods [43].

\section{$\mathrm{NaCl}$ treatment}

First, the selected 24 seedlings (12 'Qingxiang' walnuts and 12 'Xiangling' walnuts) were irrigated with $\mathrm{NaCl}-$ free $1 / 2$ Hoagland solution, until the soil surface was covered by a thin layer of water and this was maintained for $72 \mathrm{~h}$. Then, the solution of 9 seedlings of each variety was replaced with $1 / 2$ Hoagland nutrient solution containing $300 \mathrm{mM} \mathrm{NaCl}$. When the treatment time reached 6 , $12,24,48$ and $72 \mathrm{~h}$, the leaves were collected in triplicate, respectively. The remaining 3 seedlings of each variety were watered with $\mathrm{NaCl}$-free $1 / 2$ Hoagland solution and used as a control $[43,44]$.

\section{Identification and chromosomal locations of the HSF genes}

The domain architecture of the $H S F$ genes was obtained from Pfam database (http://pfam.sanger.ac.uk) [45] and their chromosomal locations in the walnut genome (Juglans microcarpa x Juglans regia) were downloaded from NCBI database (https://www.ncbi.nlm.nih.gov/gen ome/?term=txid2249226[orgn) [46]. The walnut HSF gene sequences were downloaded from iTAK (http:// itak.feilab.net/cgi-bin/itak/index.cgi) database. Then candidate walnut HSF coding region gene sequences were obtained by merged using the software UltraEdit, BLAST (blast-2.60) and HMMER searchers [47]. Conserved Domain Search (https://www.ncbi.nlm.nih.gov/Structure/ cdd/wrpsb.cgi) was applied to analyze the core domain of the candidate HSFs. ExPASy proteomics server (https://web.expasy.org/protparam/) was used to 
estimate the amino acid number, the molecular weight and the theoretical isoelectric point (PI) [43].

\section{Multiple alignments and phylogenetic analyses}

ClustalX 2.1 was used to align the protein sequences of the HSF genes [48]. The protein sequences of the HSF genes from $A$. thaliana and the walnut were used to construct a neighbor-joining tree with 1000 bootstrap replications using MEGA6 [49]. The MEME (Multiple Em for Motif Elicitation) tool (http://meme-suite.org/ tools/meme) [50] was chosen to analyze the conservative motifs. The amino acid sequence file was saved into the FASTA format and input into the online tool MEME [51]. The parameters were set as follows: the minimum length of the conservative motif is 6 , the maximum length is 50 , the maximum number of conserved motifs is 20 , and the others are default values.

\section{qRT-PCR analysis}

Total RNA was extracted from the leaf tissues of 'Qingxiang' and 'Xingling' varieties using an improved $\mathrm{CTAB}$ method [52]. First-strand cDNA was synthesized from $1 \mathrm{mg}$ DNase-treated total RNA using a mixture of Oligo dT Primer and Random 6 mers (PrimeScript ${ }^{\text {tim }}$ RT Master Mix, TaKaRa, Xi'an, Shaanxi, China) and the reaction volume was $10 \mu \mathrm{L}$ [53]. Gene-specific primers were designed and shown in Table S1. To verify the specificity of the primers, 28 genes of the 2 varieties were amplified by PCR, respectively (Figure S1). qRT-PCR analysis was conducted using TB green (TB Green ${ }^{\text {Tx }}$ Premix Ex Taq ${ }^{\text {Th }}$ II, Takara) with an IQ5 real-time PCR machine (Bio-Rad, Hercules, CA, USA). Each reaction was carried out in triplicate with a reaction volume of $20 \mu \mathrm{L}$. Cycling parameters were $95^{\circ} \mathrm{C}$ for $30 \mathrm{~s}$, followed by 40 cycles of $95^{\circ} \mathrm{C}$ for $5 \mathrm{~s}$ and $60^{\circ} \mathrm{C}$ for $30 \mathrm{~s}$. For dissociation curve analysis, a program including $95^{\circ} \mathrm{C}$ for $15 \mathrm{~s}$, followed by a constant increase from $60^{\circ} \mathrm{C}$ to $95^{\circ} \mathrm{C}$, which was included after the PCR cycles. The qRT-PCR contained three biological repeats. The 18S-RNA gene [41] was used as an internal control. The primer sequences were listed in Table S1. Relative expression levels were analyzed using the IQ5 software and the normalized expression method. The results were evaluated by the $2^{-\Delta \Delta \mathrm{CT}}$ method [54]. Details of the experimental conditions are provided in Table S2.

\section{Supplementary information}

Supplementary information accompanies this paper at https://doi.org/10. 1186/s12864-020-06879-2.

\section{Additional file 1: Figure S1. PCR analysis of JrHSFs in 'Qingxiang' and} 'Xianging'.

Additional file 2: Table S1. Gene-specific primers of JrHSFs and 18SRNA gene.

Additional file 3: Table S2. qRT-PCR relative expression data of Fig. 5 .

\section{Abbreviations}

HT: High temperature; HSR: Heat shock response; HSFs: Heat stress transcription factors; DBD: DNA-binding domain; OD or HR-A/ B: Oligomerization domain; NLS: Nuclear localization signal; NES: Nuclear export signal; CTD: C-terminal domain; HSE: Heat shock element; HSPs: Heat shock proteins; AHA: Activator motif; TFs: Transcription factors; SA: Salicylic acid; qRT-PCR: quantitative Real-time PCR; PI: Isoelectric point; WAK: Wallassociated kinase; NBS-LRR: Nucleotide binding site-leucine-rich repeat; SAURs: Small auxin-up RNAs; HB: Homeobox; SAR: System-acquired heat resistance; GolS1: Galactinol synthase; ABA: Abscisic acid; AREs: ABA response elements; ROS: Reactive Oxygen Species

\section{Acknowledgements}

Not applicable.

\section{Authors' contributions}

$X J L$ and SBP designed and wrote the paper; XJL, PPM, and MYZ did all the experiments; MZZ conducted data analysis; SBP and GYY checked the data analysis and revised the Paper. All authors have read and approved the manuscript.

\section{Funding}

This research was funded by the Ministry of Science and Technology of the People's Republic of China (grant number "2017YFD0600103-4-2") and National Natural Science Foundation of China (grant number "31670650").

The funding agency was not involved in the design of the study, collection, analysis, interpretation of data and writing the manuscript.

\section{Availability of data and materials}

All data generated or analyzed during this study are included in this published article [and its supplementary information files]. The planting area and yield of Walnut in China in 2017 were obtained from FAO (http://www. fao.org/faostat/en/\#data/QC/visualize). The domain architecture of the HSF genes was obtained from Pfam database (http://pfam.sanger. ac.uk). The chromosomal locations in the walnut genome (Juglans microcarpa $x$ Juglans regia) were downloaded from $\mathrm{NCBI}$ database (https://www.ncbi.nlm. nih.gov/ genome/?term=txid2249226[orgn). The walnut HSF gene sequences were downloaded from iTAK (http://itak.feilab.net/cgi-bin/itak/index.cgi) database. Conserved Domain Search (https://www.ncbi.nlm.nih.gov/Structure/cdd/ wrpsb.cgi) was applied to analyze the core domain of the candidate HSFs. ExPASy proteomics server (https://web.expasy.org/protparam/) was used to estimate the amino acid number, the molecular weight and the theoretical isoelectric point (PI). The MEME (Multiple Em for Motif Elicitation) tool (http:// meme-suite.org/tools/meme) was chosen to analyze the conservative motifs.

Ethics approval and consent to participate

Not applicable.

\section{Consent for publication}

Not applicable.

\section{Competing interests}

The authors declare that they have no competing interests.

Received: 23 December 2019 Accepted: 2 July 2020

Published online: 10 July 2020

\section{References}

1. Martínez-García PJ, Crepeau MW, Puiu D, Gonzalez-Ibeas D, Whalen J, Stevens KA, Paul R, Butterfield TS, Britton MT, Reagan RL, Chakraborty S, Walawage SL, Vasquez-Gross HA, Cardeno C, Famula RA, Pratt K, Kuruganti S, Aradhya MK, Leslie CA, Dandekar AM, Salzberg SL, Wegrzyn JL, Langley $\mathrm{CH}$, Neale DB. The walnut (Juglans regia) genome sequence reveals diversity in genes coding for the biosynthesis of non-structural polyphenols. Plant J. 2016;87(5):507-32.

2. Ju GH, Cui LJ, Zhang S, Qian ZY, Pan JX, Kai GY. Research progress on molecular mechanism of plant heat tolerance. J Changjiang Vegetables. 2012;24:5-11.

3. Xie H, Chong P, Li Y, Zhan J, Zhou P. Effects of exogenous nitrogen on physiological characteristics of walnut seedlings under drought stress. Mol Plant Breed. 2019;17(18):6152-60. 
4. Matsuura H, Ishibashi Y, Shinmyo A, Kanaya S, Kato K. Genome-wide analyses of early translational responses to elevated temperature and high salinity in Arabidopsis thaliana. Plant Cell Physiol. 2010;51(3):448-62.

5. Duan S, Liu B, Zhang Y, Li G, Guo X. Genome-wide identification and abiotic stress-responsive pattern of heat shock transcription factor family in Triticum aestivum L. BMC Genomics. 2019;20(1):220-57.

6. Zhang DJ, Yan JP, Chen LM. Research progress on heat shock factors of plant a. Biotechnology. 2010;3:92-5.

7. Nover L, Bharti K, Döring P, Mishra SK, Ganguli A, Scharf KD. Arabidopsis and the heat stress transcription factor world: how many heat stress transcription factors do we need? Cell Stress Chaperones. 2001;6(3):177.

8. Scharf K, Berberich T, Ebersberger I, Nover L. The plant heat stress transcription factor ( $\mathrm{Hsf}$ ) family: structure, function and evolution. BBA Gene Regul Mech. 2012;1819:104-19.

9. Damberger FF, Pelton JG, Harrison CJ, Nelson HC, Wemmer DE. Solution structure of the DNA-binding domain of the heat shock transcription factor determined by multidimensional het-eronuclear magnetic resonance spectroscopy. Protein Sci. 1994;3(10):1806-21.

10. Kotak S, Port M, Ganguli A, Bicker F, Koskull-Doring P. Characterization of Cterminal domains of Arabidopsis heat stress transcription factors ( $\mathrm{Hsfs}$ ) and identification of a new signature combination of plant class a Hsfs with AHA and NES motifs essential for activator function and intracellular localization. Plant J. 2004;39:98-112.

11. Treuter E, Nover L, Ohme K, Scharf KD. Promoter specificity and deletion analysis of three heat stress transcription factors of tomato. Mol Gen Genet. 1993;240:113-25.

12. Döring $P$, Treuter $E$, Kistner C, Lyck R, Chen A, Nover L. Role of AHA motifs for the activator function of tomato heat stress transcription factors HsfA1 and HsfA2. Plant Cell. 2000;12:265-78.

13. Nover $L$, Scharf KD. Heat stress proteins and transcription factors. Cell Mol Life Sci. 1997:53:80-103.

14. Guo M, Liu JH, Ma X, Lou DX, Gong ZH, Lu MH. The plant heat stress transcription factors (HSFs): structure, regulation, and function in response to abiotic stresses. Front Plant Sci. 2016;7:114.

15. Baniwal SK, Bharti K, Chan KY, Fauth M, Ganguli A, Kotak S, Mishra SK, Nover L, Port M, Scharf KD, Tripp J, Weber C, Zielinski D, von KoskullDöring P. Heat stress response in plants: a complex game with chaperones and more than twenty heat stress transcription factors. J Biosci. 2004;29(4):471-87.

16. Chan-Schaminet KY, Baniwal SK, Bublak D, Nover L, Scharf KD. Specific interaction between tomato HsfA1 and HsfA2 creates hetero-oligomeric superactivator complexes for synergistic activation of heat stress gene expression. J Biol Chem. 2009;284:20848-57.

17. Wang GD, Kong FY, Meng QW. Research progress of tomato heat shock transcription factor. Acta Phys Sin. 2013;3:217-24.

18. Zhang J, Jia LN, Zhao YP, Lu C, Wei N. Development and utilization of Juglans regia male inflorescences in China: research Progress. J Agric For. 2018;8(11):54-7.

19. Zhang SB, Chen C, Li L, Meng L, Singh J, Jiang N, Deng XW, He ZH, Lemaux PG. Evolutionary expansion, gene structure, and expression of the rice wallassociated kinase gene family. Plant Physiol. 2005;139(3):1107-24.

20. Bai J, Pennill LA, Ning J, Lee SW, Ramalingam J, Webb CA, Zhao B, Sun Q, Nelson JC, Leach JE, Hulbert SH. Diversity in nucleotide binding site-leucinerich repeat genes in cereals. Genome Res. 2002;12(12):1871-84.

21. Jain M, Tyagi AK, Khurana JP. Genome-wide analysis, evolutionary expansion, and expression of early auxin-responsive SAUR gene family in rice (Oryza sativa). Genomics. 2006;88(3):360-71.

22. Li Y, Zhu Y, Yao J, Zhang S, Wang L, Guo C, Steven VN, Wang X. Genomewide identification and expression analyses of the homeobox transcription factor family during ovule development in seedless and seeded grapes. Sci Rep-UK. 2017;7(1):12638.

23. Gu Y, Ji Z, Chi F, Qiao Z, Xu C, Zhang J, Dong Q, Zhou Z. Bioinformatics and expression analysis of the WRKY gene family in apple. Sci Agric Sin. 2015; 48(16):3221-38

24. Li HY, Wang FG, Qiu NW, Zhang YH, Li HY, Li JJ, Gao JW. Research progress of plant class B and C heat shock transcription factors. Shandong Agric Sci. 2015;1:144-8.

25. Liu G, Chai F, Wang Y, Jiang J, Duan W, Wang Y, Wang F, Li S, Wang L. Genome-wide identification and classification of HSF family in grape, and their transcriptional analysis under hat acclimation and heat stress. Horticult Plant J. 2018;4:133-43.
26. Xiao Z, Huang RM, Wang QZ, Li AH, Chang CF, Xu YJ. Walnut in different ecological climate zones: nut quality comparison. Chin Agric Sci Bull. 2019; 35(28):70-4.

27. Shi QH, Bao ZY, Zhu ZJ, Ying QS, Qian QQ. Effects of different treatments of salicylic acid on heat tolerance, chlorophyll fluorescence, and antioxidant enzyme activity in seedlings of Cucumis sativa L. Plant Growth Regul. 2006; 48(2):127-35.

28. Wang RH, Mei Y, Xu L, Zhu XW, Wang Y, Guo J, Liu LW. Genome-wide characterization of differentially expressed genes provides insights into regulatory network of heat stress response in radish (Raphanus sativus L.). Funct Integr Genomic. 2018;18(2):225-39.

29. Anckar J, Sistonen L. Regulation of HSFI function in the heat stress response, implications in aging and disease. Annu Rev Biochem. 1819;2011:104-19.

30. Zhang SS, Liu JX, Lu SJ. Heat shock transcription factor HSFAld is involved in ER stress response in Arabidopsis. Sci Sin Vitae. 2016; 46(04):441-8

31. Qu A, Ding Y, Jiang Q, Zhu C. Molecular mechanisms of the plant heat stress response. Biochem Bioph Res Co. 2013;432:203-7.

32. Li L, Liu SQ, Yang YH, Dai LY, LI W. Progress on the function of heat shock transcription factors in plant abiotic stress tolerance. Current Biotechnol. 2018;3:214-20.

33. Panikulangara TJ, Eggers-Schumacher G, Wunderlich M, Stransky H, Schöffl F. Galactinol synthase1 a novel heat shock factor target gene responsible for heatinduced synthesis of raffinose family oligosaccharides in Arabidopsis. Plant Physiol. 2004;136(2):3148-58.

34. Zhu JK, Ni JP. Plant abiotic stress signal transduction and response. Chin Rice. 2016;6:52-60.

35. Peng SB, Zhu ZG, Zhao K, Shi JL, Yang YZ, He MY, Wang YJ. A novel heat shock transcription factor, VpHsf1, from Chinese wild Vitis pseudoreticulata is involved in biotic and abiotic stresses. Plant Mol Biol Report. 2012;31:240-7.

36. Tenhaken R. Cell wall remodeling under abiotic stress. Front Plant Sci. 2014;5:771.

37. Liu HT, Sun DY, Zhou RG. Ca ${ }^{2+}$ and $\mathrm{AtCaM}_{3}$ are involved in the expression of heat shock protein gene in Arabidopsis. Plant Cell Environ. 2005;28:1276-84.

38. Suri SS, Dhindsa RS. A heat-activated MAP kinase (HAMK) as a mediator of heat shock response in tobacco cells. Plant Cell Environ. 2008;31:218-26.

39. Saidi Y, Finka A, Muriset M, Bromberg Z, Weiss YG, Maathuis FJ, Goloubinoff $P$. The heat shock response in moss plants is regulated by specific calcium-permeable channels in the plasma membrane. Plant Cell. 2009;21:2829-43.

40. Volkov RA, Panchuk II, Mullineaux PM, Schöffl F. Heat stress-induced $\mathrm{H}_{2} \mathrm{O}_{2}$ is required for effective expression of heat shock genes in Arabidopsis. Plant Mol Biol. 2006;61:733-46.

41. Yang G, Zhang W, Liu Z, Yi-Maer AY, Zhai M, Xu Z. Both JrWRKYZ and JrWRKYY of Juglans regia mediate responses to abiotic stresses and abscisic acid through formation of homodimers and interaction. Plant Biol. 2017; 19(2):268-78.

42. Yao W, Zhang D, Zhou B, Wang J, Li R, Jiang T. Over-expression of poplar NAC15 gene enhances wood formation in transgenic tobacco. BMC Plant Biol. 2020. https://doi.org/10.11186/s12870-019-2191-2.

43. Chen X, Chen R, Wang Y, Wu C, Huang J. Genome-Wide Identification of WRKY Transcription Factors in Chinese jujube (Ziziphus jujuba Mill.) and their Involvement in Fruit Developing, Ripening, and Abiotic Stress. Genes. 2019;10(5):360.

44. Yao W, Zhou B, Zhang X, Zhao K, Cheng Z, Jiang T. Transcriptome analysis of transcription factor genes under multiple abiotic stresses in Populus simonii × P. nigra. Gene. 2019;707:189-97.

45. Finn RD, Mistry J, Tate J, Coggill PC, Heger A, Pollington JE, Gavin OL, Gunasekaran P, Ceric G, Forslund K, Liisa Holm L, Sonnhammer ELL, Eddy SR, Bateman A. The Pfam protein families database. Nucleic Acids Res. 2010; 38:211-22.

46. Zhu T, Wang L, You FM, Rodriguez JC, Deal KR, Chen L, Li J, Chakraborty S, Balan B, Jiang CZ, Brown PJ, Leslie CA, Aradhya MK, Dandekar AM, MCGuire PE, Kluepfel D, Dvorak J, Luo MC. Sequencing a Juglans regia $\times J$. Microcarpa hybrid yields high-quality genome assemblies of parental species. Hortic Res-England. 2019;6(1):16-55.

47. Camacho C, Coulouris G, Avagyan V, Ma N, Papadopoulos J, Bealer K, Madden TL. BLAST+: architecture and applications. BMC Bioinformatics. 2009;10:421

48. Larkin MA, Blackshields G, Brown NP, Chenna R, McGettigan PA, McWilliam H, Valentin F, Wallace IM, Wilm A, Lopez R, Thompson JD, 
Gibson TJ, Higgins DG. Clustal W and Clustal X version 2.0. Bioinformatics. 2007;23(21):2947-8.

49. Tamura K, Stecher G, Peterson D, Filipski A, Kumar S. MEGA6: molecular evolutionary genetics analysis version 6.0. Mol Biol Evol. 2013;30(12):2725-9.

50. Bailey TL, Elkan C. The value of prior knowledge in discovering motifs with MEME. Proc Int Conf Intell Syst Mol Biol. 1995;3:21-9.

51. Timothy $L B$, Charles E. Fitting a mixture model by expectation maximization to discover motifs in biopolymers. Proc Int Conf Intell Syst Mol Biol. 1994;2:28-36.

52. Wang J, Wang Q, Tian N, Wang Y, Wang AX, Zhang KZ, Cui JT. Comparative and analysis of RNA extracting methods from different plant tissues. J Beijing Univ Agric. 2015;30(1):76-80.

53. Zhang YC, Gao M, Singer SD, Fei ZJ, Wang H, Wang XP. Genome-wide identification and analysis of the TIFY gene family in grape. PLoS One. 2012:7:e44465.

54. Livak KJ, Schmittgen TD. Analysis of relative gene expression data using real-time quantitative $P C R$ and the $2^{-\Delta \Delta C T}$ method. Methods. 2001;25:402-8.

\section{Publisher's Note}

Springer Nature remains neutral with regard to jurisdictional claims in published maps and institutional affiliations.

Ready to submit your research? Choose BMC and benefit from:

- fast, convenient online submission

- thorough peer review by experienced researchers in your field

- rapid publication on acceptance

- support for research data, including large and complex data types

- gold Open Access which fosters wider collaboration and increased citations

- maximum visibility for your research: over $100 \mathrm{M}$ website views per year

At BMC, research is always in progress.

Learn more biomedcentral.com/submissions 